\title{
Visión y perspectiva del ingeniero industrial en la gestión de organizaciones
}

\section{RESUMEN}

En el proceso de desarrollo de la ingeniería industrial se ha verificado la importancia de la administración cientifica para el crecimiento de las empresas, coadyuvando a la consolidación de los sectores industriales y al desarrollo de las naciones, cumpliendo el objetivo de Taylor del desarrollo económico a través de la eficiencia empresarial. A pesar que los ingenieros industriales presentan un elevado enfoque en los resultados y la mejora de las organizaciones, no alcanzan el éxito de otras profesiones, enfocadas en temas no empresariales.

El estudio exploratorio tiene el objetivo de identificar preliminarmente, los elementos por los cuales, ante requerimiento para ocupar las posiciones directivas de alto nivel en organizaciones, se tiende a elegir a las profesiones humanisticas, como los abogados, antes que las profesiones empresariales, como los ingenieros industriales.

Se han identificado los elementos que delimitan la preferencia señalada. Estos son: a) método de solución analítico-reduccionista frente a historicista-integrador, b) enfoque convergente y enfocado en temas concretos frente a enfoque divergente y amplio, c) resultados definidos como logros concretos a corto plazo frente a logros de largo plazo, d) jerarquía de problemas con recursos y variables tangibles de nivel operativo frente a conceptos $y$ variables sociales de nivel estratégico.

Palabras clave: Status y rol profesional, jerarquia de problemas, visión, misión profesional.

VISION AND PERSPECTIVE OF INDUSTRIAL ENGINEERING IN MANAGEMENT OF ORGANIZATIONS

ABSTRACT

In the process of development of industrial engineering, it has been verified the importance of scientific management for business growth, contributing to the consolidation of the industrial clusters and developing nations, reaching Taylor's target for economic development through business efficiency. Although industrial engineers have a high focus on results and improve of organizations, do not reach the success of other professions, which are focused on nonbusiness topics.

The exploratory study aims to identify preliminarily, elements which, in front of requirement to occupy senior management positions in organizations, the stockholders tend to elect humanistic professions, such as lawyers, before the business professions such as industrial engineers.

They have been identified the elements that define the preference stated. These are: a) method o solution analyticalreductionist against historicist-integrator, b) focus convergent approach on specific issues against divergent and wide approach, c) achievement as specific outcomes defined as short-term gains against long-term gains, d) hierarchy of problems with tangible resources and operational level variables versus social concepts and strategic level variables.

Keywords: Status and professional role, hierarchy of problems, vision, professional mission.

\section{ANTECEDENTES DEL ESTUDIO}

El objetivo del presente artículo es describir los elementos que diferencian a los ingenieros industriales orientados a la competitividad, frente a otras profesiones orientadas a posiciones, los que acceden niveles directivos diferentes al del ingeniero industrial. Estos elementos se han identificado mediante un estudio de investigación realizado durante el año 2011, cuyos hallazgos se consideran en el presente artículo.

\subsection{Antecedentes filosóficos}

Bajo el enfoque de sistemas, el tema es la manera en que se clasifican las profesiones a través de sus semejanzas y diferencias. Entre sus semejanzas se encuentran los esquemas de formación orientados hacia una comunidad académica de conocimiento, entre las diferencias se encuentran las prioridades que los demandantes de altos directivos (gobiernos, empresas) realizan entre una u otra profesión. Para las diferencias, se considera el esquema de prioridades de la sistemática aristotélica por la cual un sistema natural puede definirse como uno en que las cosas pueden colocarse en grupos apropiados mediante la referencia a un número de características, correspondientes a la esencia por el cual se conforman los grupos.

\subsection{Antecedentes académicos}

Los antecedentes académicos se sintetizan en el concepto de sistemas de actividad humana desarrollado por teóricos de la Ingeniería de sistemas industriales ${ }^{4}$, que afirman que la Ingeniería Industrial se encarga del diseño, planeación implantación y manejo de sistemas de actividad humana, o sea, la creación y gestión de sistemas de producción conformados por personas y cosas (máquinas, materiales, ambiente), empleando herramientas técnicas orientadas a elevar la productividad, mejorar la eficiencia, lograr la mayor eficacia y resolver problemas empresariales complejos.

1 Profesor en la Facultad de Ingeniería Industrial, Departamento de Producción y Gestión Industrial de la UNMSM.

E-mail: orestescachay@yahoo.es

2 Profesor en la Facultad de Ingeniería Industrial, Departamento de Producción y Gestión Industrial de la UNMSM.

E-mail: aacevedo@speedy.com.pe

3 Profesor en la UPG de la Facultad de Ingeniería de Sistemas e Informática de la UNMSM. E-mail: klinares@speedy.com.pe

4 Ídem 


\section{EL MARCO CONCEPTUAL DEL ESTUDIO}

Las profesiones conforman comunidades del saber, orientadas, por un lado al aprendizaje de técnicas y herramientas empíricas y por otro lado, a la creación de conocimiento mediante la investigación y desarrollo de teorías y modelos.

\subsection{El rol y alcance de las profesiones}

Las profesiones poseen un status en función al rol que ejercen dentro de la sociedad. Las profesiones de mayor amplitud poseen mayor capacidad para influir, en la orientación del uso de recursos o en la dirección de personas. En la figura 1 se muestra que las profesiones orientadas a las cosas físicas poseen influencia en ámbitos restringidos y espe- cializados, en cambio las profesiones orientadas a los aspectos sociales y humanos, poseen mayor influencia (por ejemplo, un vocal supremo de la Corte de Justicia posee mayor visibilidad e influencia , que un investigador en energía nuclear del IPEN).

El ingeniero industrial se encarga de sistemas de transformación, maneja materias primas y maneja recursos humanos para transformar insumos en productos. Se ubica en el justo medio entre el investigador de las ciencias físicas y el licenciado en Derecho. Su relación con otras profesiones como el administrador o economista, se asemeja en su finalidad, por lo que el estudio se enfoca en diferenciar al ingeniero industrial frente al abogado, como casos extremos y representativos.

Figura 1. El continuo de las profesiones. Su rol y alcance

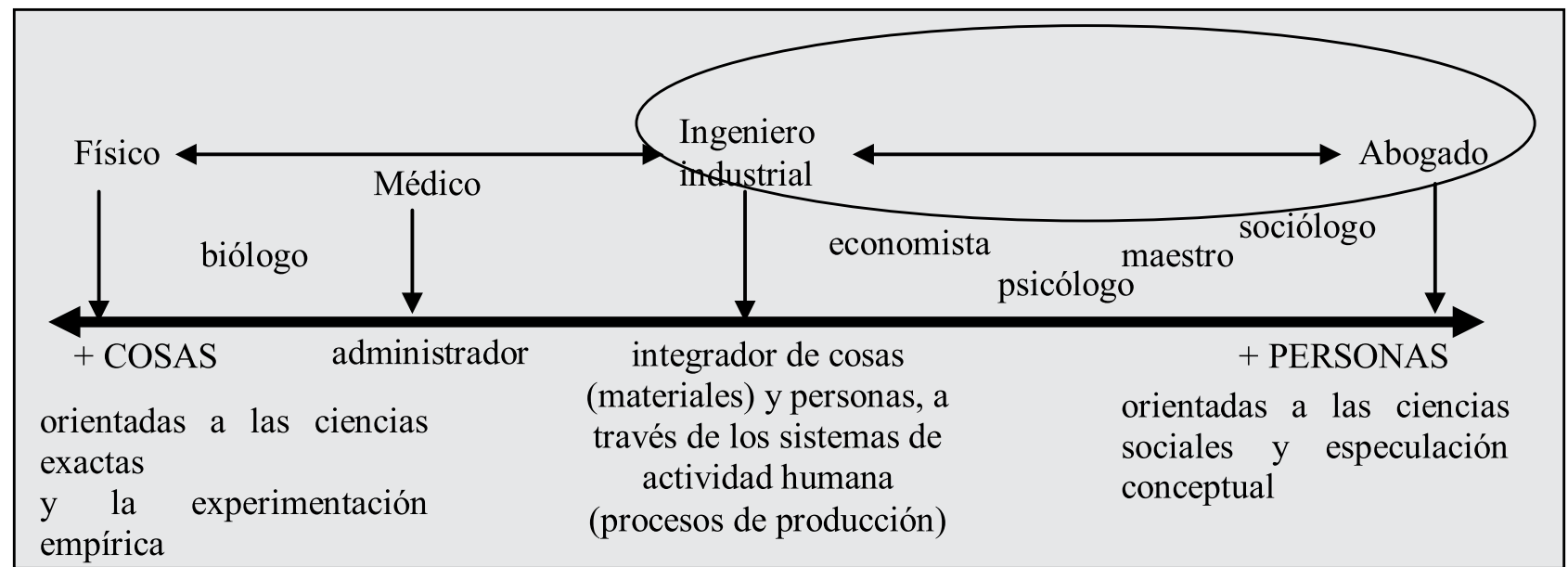

Fuente: Elaboración propia

\subsection{Los elementos de influencia profesional}

Existen elementos que permiten que una profesión, plantee una visión que facilita un posicionamiento y una mayor influencia frente al mercado de los demandantes de profesionales (empresas y gobierno). Estos elementos se muestran en la figura 2.

- La perspectiva del tiempo, identifica el momento histórico en que esta profesión se manifiesta en la sociedad y la manera en que ha evolucionado.

- Resolver situaciones, la manera en que toma decisiones y las herramientas que emplea para analizar e indagar los problemas.

- Aspecto humano sobre cual es su rol frente a la sociedad y los sistemas sociales.

- Manejo de los recursos, su responsabilidad en resultados.

\section{LA INVESTIGACIÓN CUALITATIVA DE LAS PROFESIONES}

Las profesiones son parte de comunidades del saber, orientadas, por un lado al aprendizaje de técnicas y herramientas empíricas y por otro lado, a la creación de conocimiento mediante la investigación y desarrollo de teorías y modelos. Los elementos de influencia, se han definido mediante entrevistas con especialistas.

\subsection{Perspectiva de tiempo}

El desarrollo de ambas profesiones se presenta En la figura 3. Se diferencian en los términos empleados: Derecho se refiere a sociedad y paz social, ingeniería se enfoca en trabajo y métodos para mejorar la eficiencia de empresa. Ver la figura 4.

Respecto al origen de la profesión, ingeniería industrial reconoce a Taylor y fines del 1800 como 
época en que surge la administración científica, los abogados afirman que su profesión surge con la separación del Derecho y la Moral, según Mateo en el Nuevo Testamento ${ }^{5}$.

\subsection{Toma de decisiones}

El Derecho no se enfoca en resolver problemas, sino en mantener la operacionalidad de la sociedad, en conjunto. Bajo el concepto de justicia aplica criterios derivados del precedente o de la jurispru- dencia busca opciones equitativas a las que denomina casos de justicia: sus técnicas son negociables, en el sentido que se sustenta en intereses en conflicto de personas.

El Ingeniero Industrial toma decisiones busca resolver situaciones basado en experiencia, técnicas de optimización como Investigación Operativa, Análisis de Tiempos, Análisis de Decisiones, Automatización, de manera que se mejore tiempos, eficiencia y productividad.

Fioura 2. Elementos nara el nosicionamiento de una nrofesión

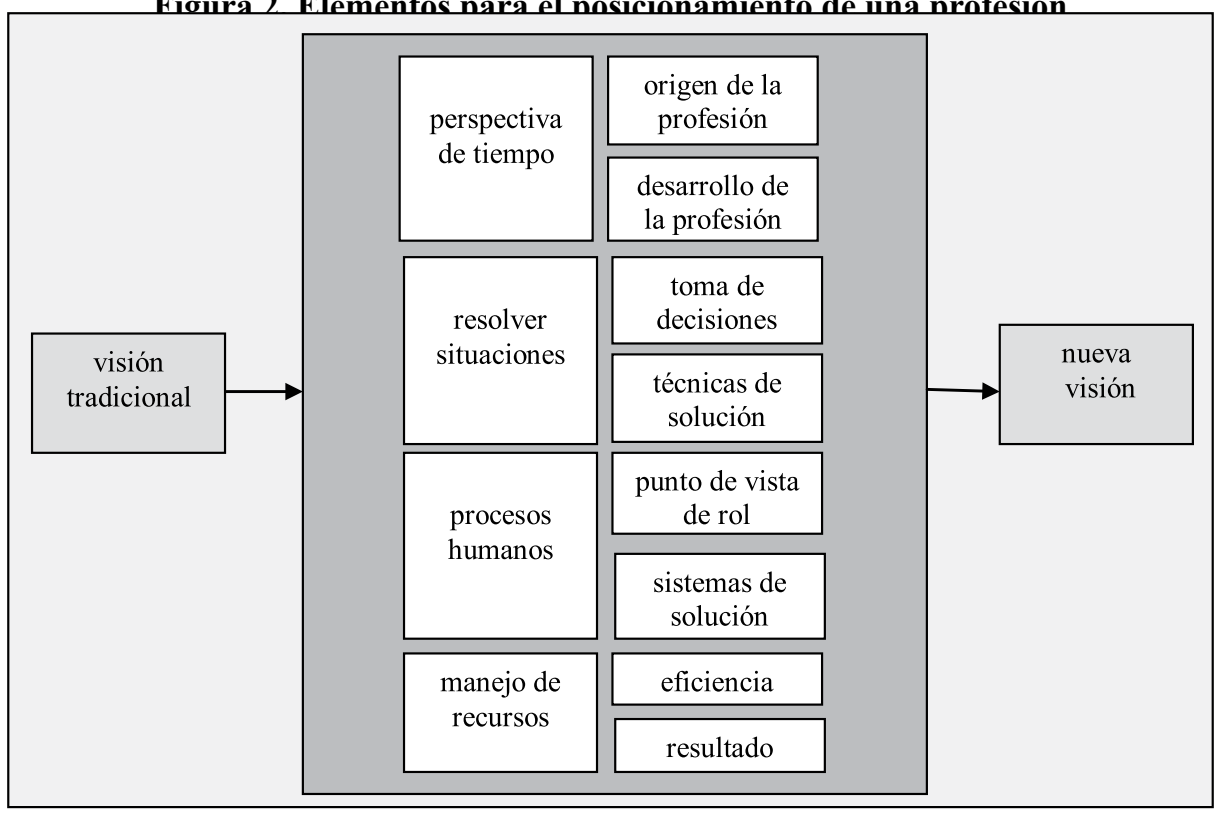

Fuente: Elaboración propia

Figura 3. Devenir en la profesión de Derecho

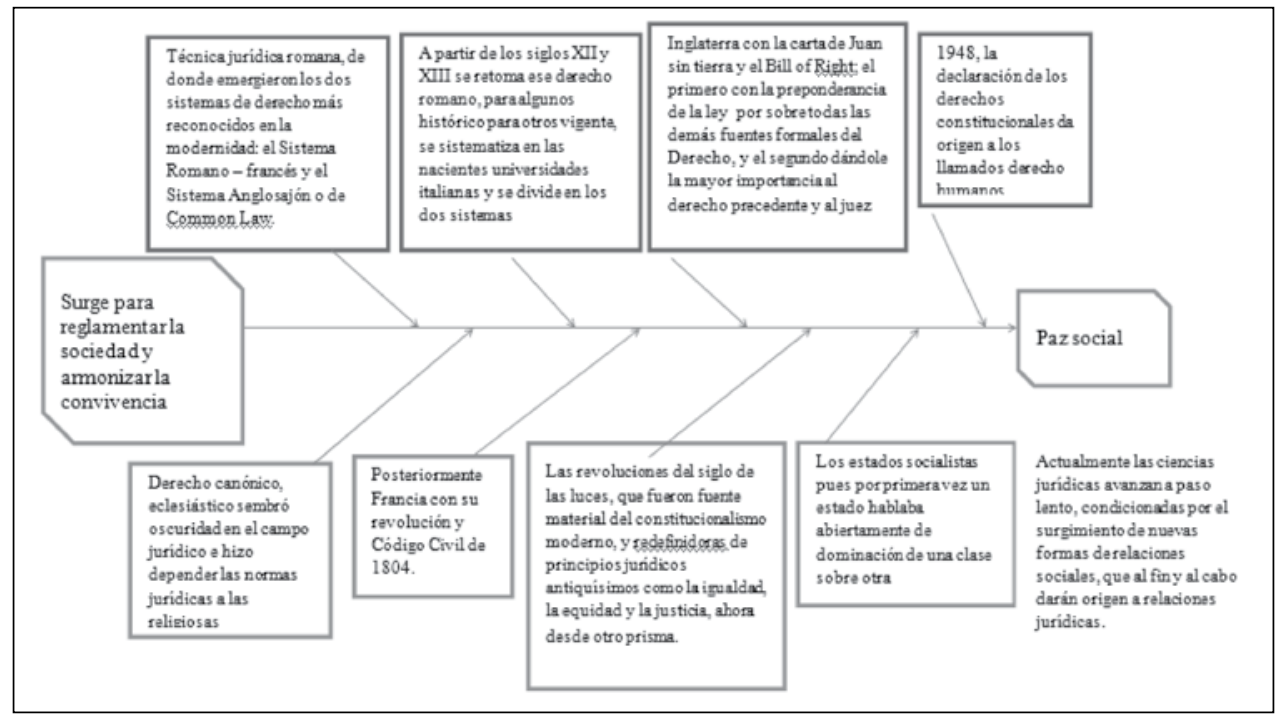

Fuente: Elaboración propia

1 "Dad al César lo que es del César y a Dios lo que es de Dios". (Mt. 22, 15-21). 
Figura 4. Devenir en la profesión de Ingeniería Industrial

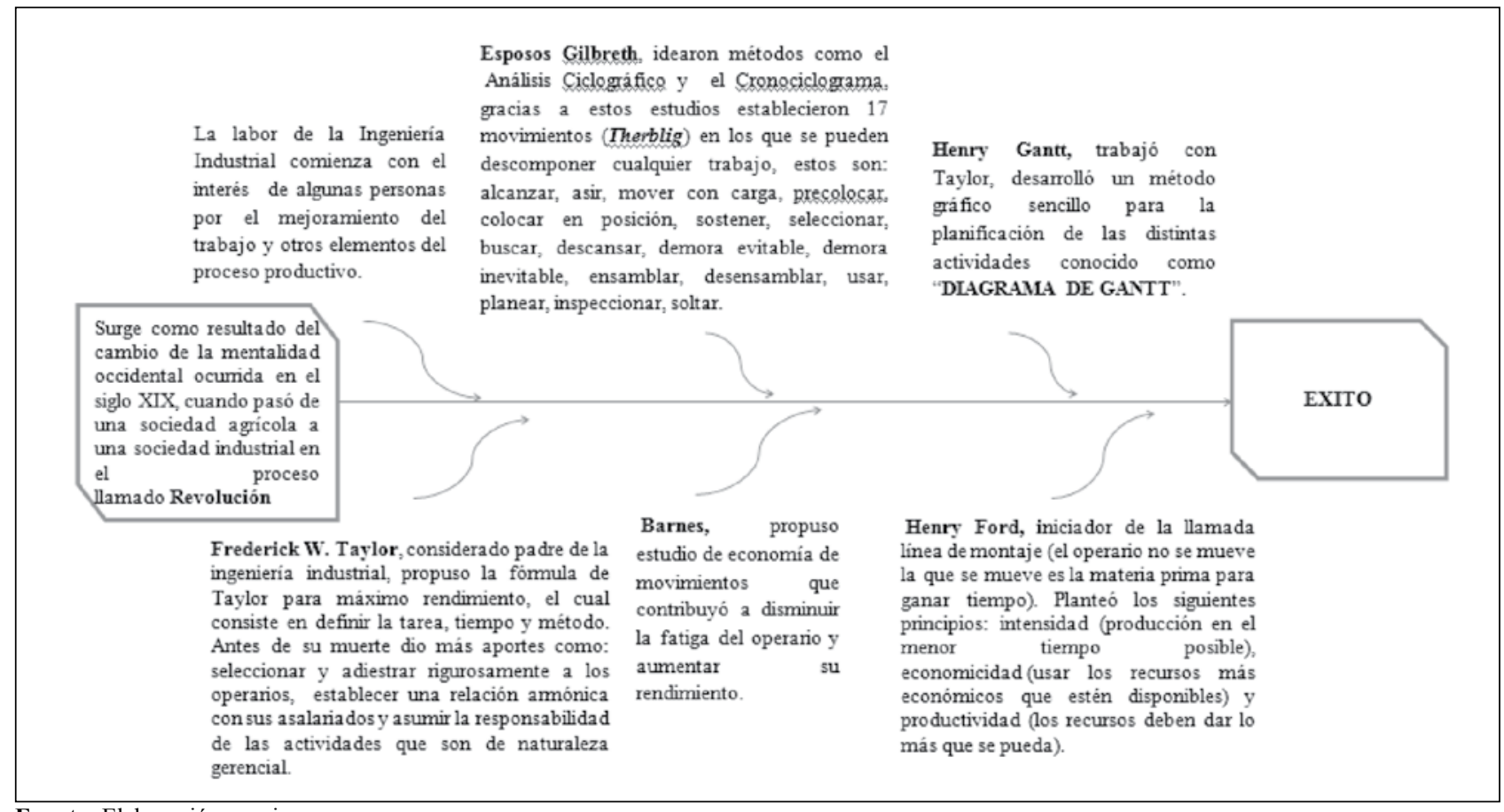

Fuente: Elaboración propia

\subsection{Los procesos de actividad humana}

Cada profesión plantea una situación previa a superar y una situación posterior que es la visión a alcanzar sistemas de solución.

En el caso de Derecho, el enfoque de la tarea a realizar (misión) se refiere a las relaciones sociales y se encuentra en la figura 5, cuyas variables relevantes son sociedad, respeto, justicia, valores, conciencia social, entre otros. En el ingeniero industrial, la tarea o misión se refiere a desarrollo y medio ambiente mediante los proceso de transformación.

Desde el punto de vista de rol que asume cada profesión, el ingeniero plantea un esquema de tipo horizontal donde las más importantes son las funciones y las tareas concretas que se realizan en las diversas áreas de la empresa. Los tres niveles clásicos de la gestión empresarial interna (operativo, táctico y estratégico) es superado por los niveles de resolución de la figura 6 .

El abogado plantea su rol de diferente manera, considerando que los resultados no son parte de su responsabilidad profesional, se concentra en ubicar las posiciones verticales, o sea no enfoca tareas a realizar sino posiciones jerárquicas a cubrir.

\subsection{La visión y la misión}

\section{- La misión de la Ingeniería Industrial}

Visión. Con los enfoque de mejora y calidad, las empresas han de requerir en su plana directiva a ingenieros industriales, dirigiendo o supervisando sus procesos, incluso en sectores de gobierno y organizaciones de servicio como seguridad social.

Misión. Ser una comunidad de conocimiento que lidere la formación de profesionales competentes, comprometidos y promotores del desarrollo de sectores industriales, grupos sociales y zonas geográficas. Formar integralmente a los profesionales en áreas de la ciencia, la tecnología y la gestión industrial, constituyéndose en generadores de conocimiento, promotores del desarrollo y aplicación de técnicas de la especialidad. Desarrollar estudios e investigaciones que generen valor a las personas, la empresa y la sociedad, dentro de principios éticos, excelencia profesional y calidad.

\section{- La misión del Derecho}

Visión del Derecho. Es la institución de formación de profesionales comprometidos con los intereses y derechos de la colectividad, con conciencia social, crítica y constructiva.

Misión. Su rol es formar expertos en el conocimiento, creación y aplicación de las normas jurídicas. Las normas han de ser el instrumento racional de ordenación de las relaciones sociales y económicas entre el Estado y los ciudadanos y las personas entre sí, con el fin de lograr en la sociedad con valores como el orden, la seguridad, la justicia y la libertad, para el bienestar de la comunidad nacional e internacional. 
Figura 5. El proceso de la profesión de Derecho e Ingeniería Industrial:

El proceso de la profesión del Derecho:

\begin{tabular}{|c|c|c|}
\hline Situación Inicial & Proceso: Misión & Salida: Visión \\
\hline $\begin{array}{l}\text { El problema de las } \\
\text { divisiones sociales, } \\
\text { existia un conflicto } \\
\text { entre los interese de } \\
\text { estas sociedades que } \\
\text { querian alcanzar sus } \\
\text { objetivos. Era } \\
\text { necesario } \\
\text { establecimiento de las } \\
\text { relaciones sociales, } \\
\text { vitales para el } \\
\text { desarrollo de la }\end{array}$ & $\begin{array}{l}\text { "Lograr el respeto y } \\
\text { cumplimiento de la Justicia } \\
\text { y de los justiciables, pues } \\
\text { tiene la obligación de } \\
\text { defender sus derechos y } \\
\text { libertades en forma eficaz y } \\
\text { oportuna de los intereses } \\
\text { juridicos de sus } \\
\text { patrocinados" }\end{array}$ & $\begin{array}{l}\text { "Liderar y alcanzar } \\
\text { los objetivos de sus } \\
\text { patrocinados } \\
\text { comprometiendo los } \\
\text { intereses y derechos } \\
\text { de la colectividad, } \\
\text { con una conciencia } \\
\text { social, crítica y } \\
\text { constructiva." }\end{array}$ \\
\hline
\end{tabular}

El proceso de la profesión Ingeniería Industrial:

Situación Inicial

Existía un cambio de la mentalidad occidental en el siglo XIX, cuando pasó de una sociedad agricola a una sociedad industrial en el proceso llamado REVOLUCIÓN INDUSTRIAL.

\begin{tabular}{|l|}
\hline $\begin{array}{l}\text { Proceso: Misión } \\
\text { "Contribuir con el } \\
\text { desarrollo sostenible } \\
\text { de la sociedad y la } \\
\text { preservación del } \\
\text { medio ambiente" }\end{array}$ \\
\hline
\end{tabular}

\section{Salida: Visión}

"Ser una Carrera líder formando profesionales líderes en dirección y gestión de procesos con habilidades técnicas y humanas, comprometidas con el desarrollo"

Figura 6. La jerarquía de problemas en organizaciones

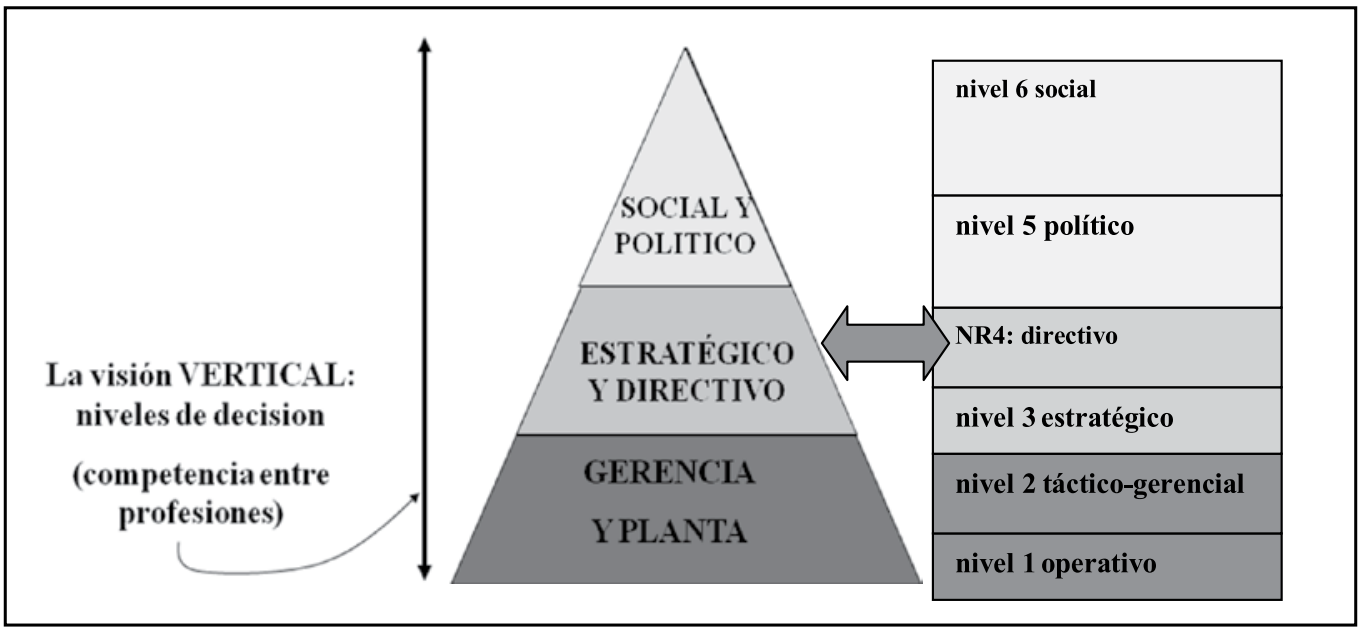

Fuente: Elaboración propia 


\section{HALLAZGOS DE LA INVESTIGACIÓN CUAN- TITATIVA}

\subsection{El Problema y pregunta de investigación}

El ingeniero industrial es un profesional orientado a obtener resultados. A pesar de los logros en empresa, frecuentemente se observa que los profesionales de otras profesiones, no orientadas a logro y resultados, poseen mayor capacidad de acceso a los puestos directivos y de alta dirección.

Entonces, la pregunta de investigación inicial es:

¿Cuáles son las razones para la diferente demanda de profesionales, para ocupar los niveles directivos mas altos, de empresa o de gobierno?.

\subsection{La hipótesis de investigación}

La Hipótesis general $\mathrm{HO}$ es: "Los egresados de Ingeniería Industrial y de Derecho manifiestan igual perspectiva en el proceso de identificar y resolver los problemas en las organizaciones".
Hipótesis alterna $\mathrm{H} 1$ es "Los egresados de Ingeniería Industrial y de Derecho manifiestan diferente perspectiva para identificar y resolver los problemas en las organizaciones".

\subsection{Análisis y discusión de los resultados}

Los resultados del estudio para muestras de ingenieros y abogados indican que, dentro de cada grupo la elección del nivel de resolución no es uniforme. La figura 7 a señala que los ingenieros se enfocan en el nivel 1 y 2 de la jerarquía de problemas (operacion y gerencia), la figura $7 \mathrm{~b}$ señala que los abogados se enfocan en el nivel 4,5 y 6 , (personas y aspectos políticos).

La figura 12 muestra el perfil de orientación a los problemas En a) los ingenieros se corrobora que se concentran en el nivel 1 y 2 , referido a manejo de los recursos de empresa, en b) los abogados se concentran en el nivel 4 y 5 , y poco en el 6 , lo que confirma su preferencia por las relaciones sociales y los temas políticos.

Figura 7. Foco de los ingenieros y los abogados

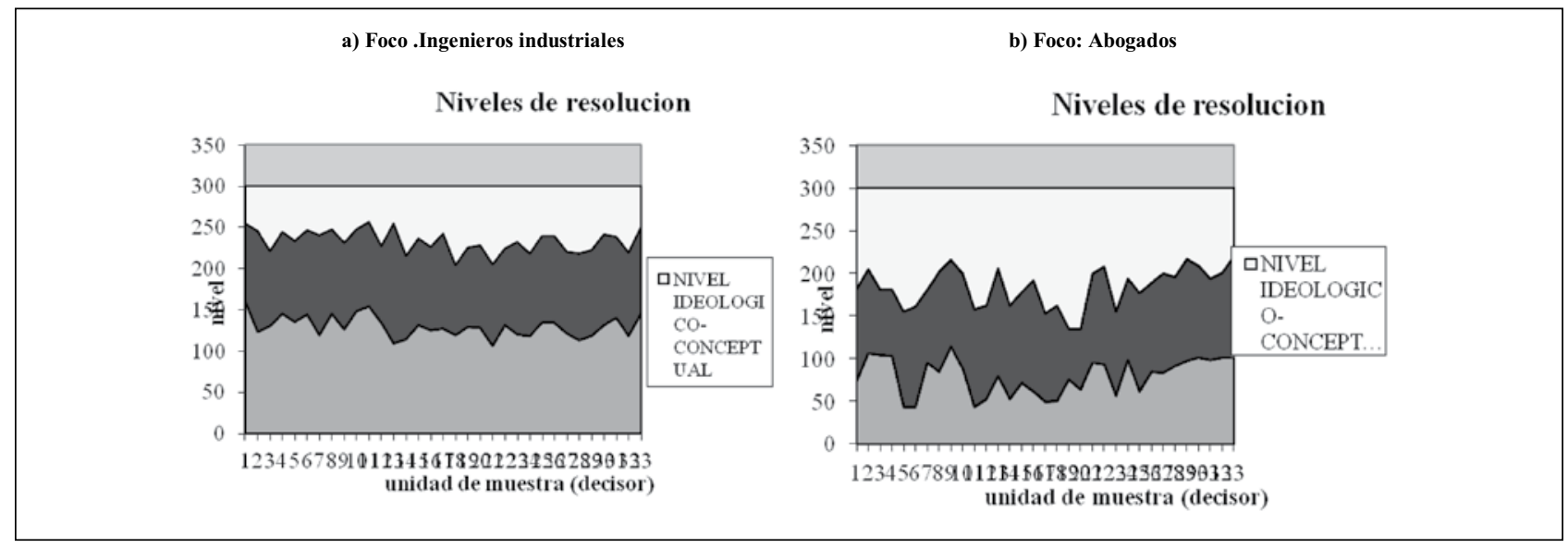

Fuente: Elaboración propia

Figura 12. La curva de frecuencia de niveles de decision

a)Curva de frecuencia de niveles de decision- Ingenieros industriales

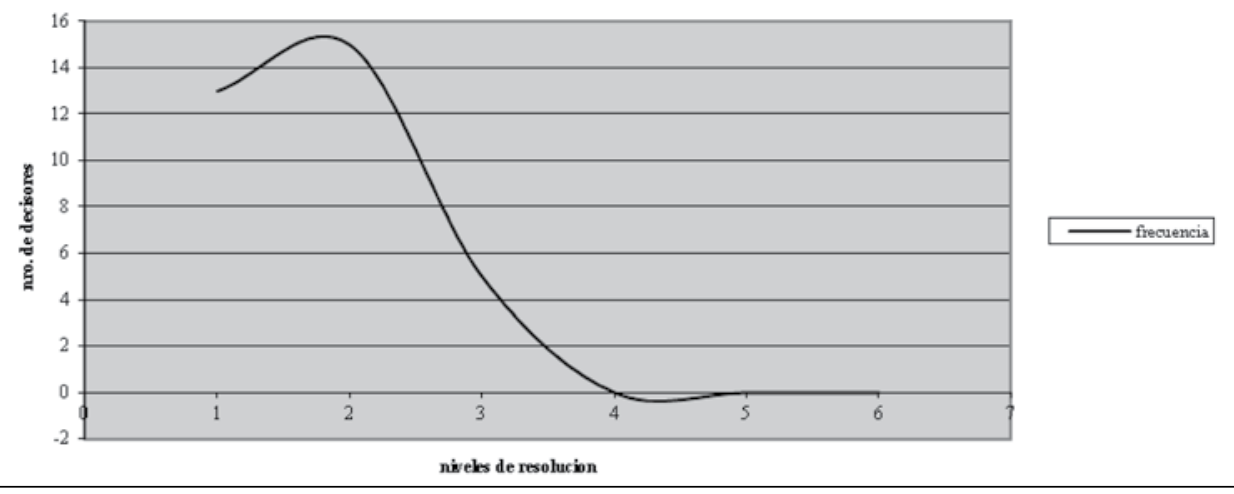




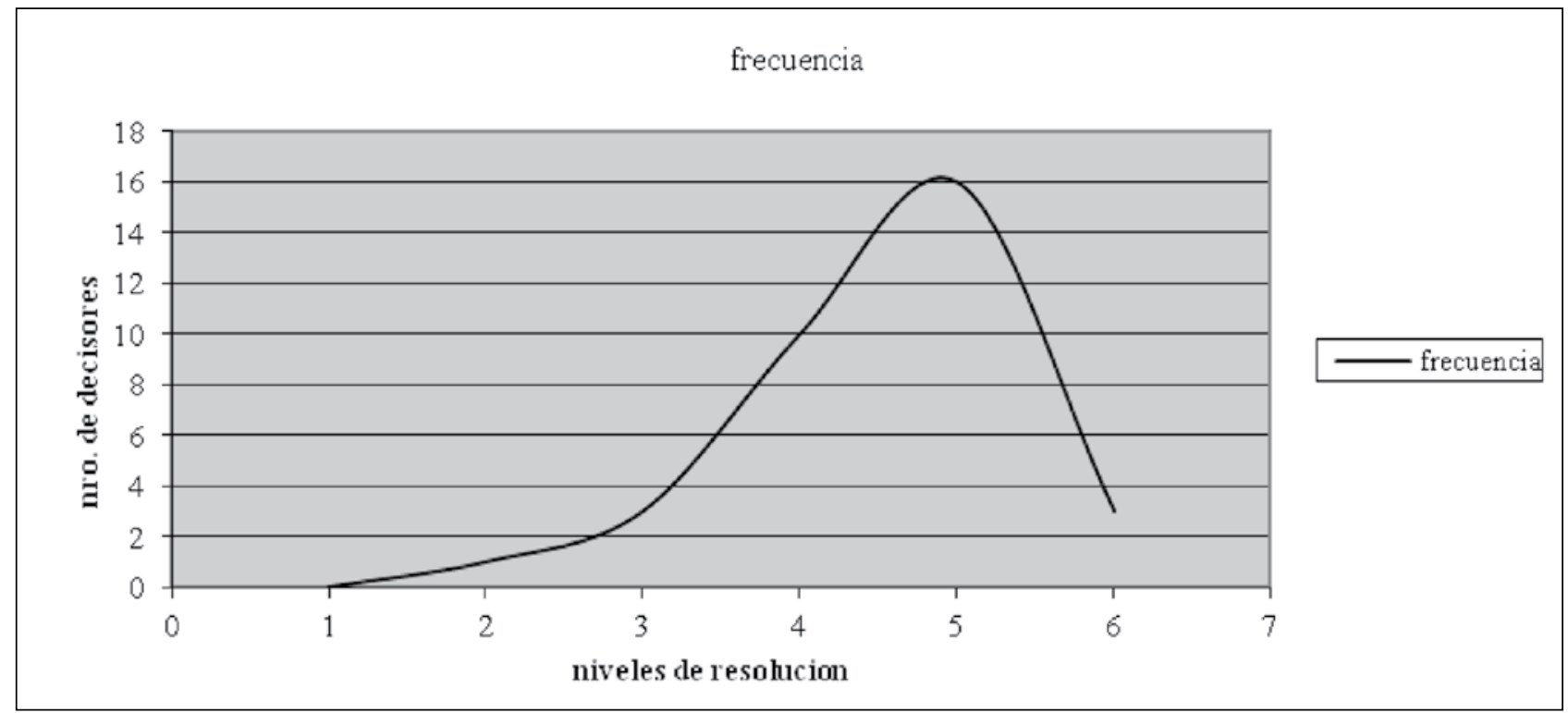

El foco concreto en la preferencia de ambas profesiones, se muestra en la figura 8 que representa la visión operativa o estratégica para ubicar problemas y afrontarlos, de una u otra profesión.. La parte a señala que los ingenieros se orientan a la operación, la parte b señala que los abogados tiene intereses más estratégicos y políticos.

\section{CONCLUSIONES DEL ESTUDIO}

El presente estudio representa un importante aporte exploratorio, cuya eficacia ha de comprender la futura profundización de los temas estudiados. A nivel de Facultad de Ingeniería Industrial, el estudio brinda elementos de juicio e información para el permanente proceso de cambio y mejora de los criterios de desarrollo profesional, la grilla curricular y de los contenidos, lo que permitirá consolidar o redefinir la orientación estratégica de largo plazo del perfil deseable del egresado de ingeniería industrial. A nivel de sociedad, el estudio plantea los elementos comparados, que han de coadyuvar hacia un perfil de ingeniero industrial mejor preparado con mayor capacidad directiva y estratégica, con superior capacidad de resolución de problemas, La adecuada asunción y desarrollo de estos elementos, contribuirá al desarrollo de las capacidades productivas y el consiguiente proceso de crecimiento de los diferentes sectores económicos.

El estudio compara a dos profesiones disímiles, los ingenieros industriales y los abogados. Por un lado, los egresados de Ingeniería Industrial poseen un enfoque analítico en el nivel jerárquico operativo donde se resuelven los problemas. La perspectiva del ingeniero industrial, los orienta hacia temas de gestion interna de las organizaciones, mientras los abogados se concentran en la viabilidad social y el manejo de los aspectos humanos.

De acuerdo al estudio, los elementos que diferencian a ambas profesiones son los siguientes:

1. Método de solución: los ingenieros industriales emplean la metodología analítica y reduccionista de la administración científica, aplicable a los problemas operativos de organizaciones, en cambio los abogados presentan emplean metodologías historicistas e integradoras, aplicable a los problemas de interrelación humana, en diferentes ámbitos de la actividad humana.

2. Enfoque: Los ingenieros industriales son convergentes y enfocados en temas concretos y focalizados cuando analizan y resuelven problemas empresariales, mientras que los abogados son divergentes y de amplia visión, son más dispersos ya que abarcan la totalidad de las actividades y niveles de los problemas de las personas.

3. Resultados: los ingenieros industriales plantean alternativas de solución donde se obtienen logros concretos. Los abogados plantean opciones donde los logros son a mediano o largo plazo, enfatizando la funcionalidad antes que los resultados concretos y de corto plazo.

4. Jerarquía de problemas: los ingenieros industriales manejan recursos y variables tangibles en el nivel operativo de las organizaciones, los abogados se dirigen al manejo de conceptos y especulación teórica sobre variables sociales en el nivel estratégico y político de las organizaciones. 


\section{REFERENCIAS}

[1] Ackoff, Russell (2000). Recreación de las corporaciones. Un diseño organizacional para el siglo XXI. Ed. Oxford University Press. México.

[2] Blair \& Whitson (1973). "Elementos de Ingeniería de Sistemas Industriales". Editorial Prentice-Hall Internacional. España.

[3] Aristóteles (1998). "Ética a Nicómaco". Editorial ALBA. España.

[4] Platón (2003). "Diálogos. Obra completa". Editorial Gredos. España.

[5] Hume, David (2005). "Investigación sobre el Conocimiento Humano". 2da. edición. Mestas Ediciones Escolares. España.

[6] Morín, Edgar (1999). "Los siete saberes necesarios para la educación del futuro". UNESCO. Francia.

[7] McGregor, Douglas (1970). "El aspecto humano de la empresa". Ed. Diana. México.

[8] Drucker, Peter (1995). "El Ejecutivo Eficaz" Editorial: Sudamericana. Colombia.

[9] Handy, Charles (1997). "Encontrar sentido en la incertidumbre", en "Repensando el futuro". Editado Rowan Gibson. Colombia.
[10] Kolb, Rubin y MacIntyre (1977). "Psicología de las Organizaciones: Problemas contemporáneos". Prentice/Hall Internacional. España.

[11] Senge, Peter (1994). "La Quinta disciplina en la práctica”. Granica. España.

[12] Vaill, Peter (1967). "La Ingeniería Industrial y los Sistemas Sociotécnicos", en Sistemas de Producción. Editado por Groff y Muth. Ed. El Ateneo, Buenos Aires.

[13] García, Teonila (2003). "Estimulación de la Creatividad en la Facultad de Ingeniería Industrial para el Desarrollo y la Producción de Software" en Industrial Data Vol.6 № 1 agosto.

[14] García, Vergiu, Párraga y Santos (2007). "Desarrollo de un modelo Multifactorial y Dinámico para la medición de los intangibles de empresas de manufactura" en Industrial Data Vol.10 No 1 enero-junio.

[15] Carrión, Rolando (2008). "Ingeniería Industrial y Desarrollo" en Industrial Data Vol.11 No 1 enero-junio. 